\title{
Determination of the Thermal Diffusivity of Plasmonic Nanofluids Containing PVP-Coated Ag Nanoparticles Using Mode-Mismatched Dual-Beam Thermal Lens Technique
}

\author{
C. S. Lopes, ${ }^{1}$ V. M. Lenart, ${ }^{2}$ R. F. Turchiello, ${ }^{2}$ and S. L. Gómez $\mathbb{D}^{1}$ \\ ${ }^{1}$ Physics Department, State University of Ponta Grossa, Ponta Grossa, PR, Brazil \\ ${ }^{2}$ Physics Department, Federal University of Technology of Paraná, Ponta Grossa, PR, Brazil \\ Correspondence should be addressed to S. L. Gómez; sgomez@uepg.br
}

Received 23 August 2018; Accepted 31 October 2018; Published 2 December 2018

Academic Editor: Joseph S. Poon

Copyright @ 2018 C. S. Lopes et al. This is an open access article distributed under the Creative Commons Attribution License, which permits unrestricted use, distribution, and reproduction in any medium, provided the original work is properly cited.

\begin{abstract}
Plasmonic nanofluids have found applications in many fields, for example, coolants, solar collectors, and theranostics agents. For such applications, an important parameter is the thermal diffusivity. In this communication, we present an experimental study concerning the dependence of the thermal diffusivity of highly diluted aqueous plasmonic nanofluids containing PVP-coated silver nanoparticles on the concentration. Measurements were made by employing the time resolved mode-mismatched thermal lens technique and the results show an enhancement of the thermal diffusivity, when compared to that of the carrier fluid, on increasing the number density of the nanoparticles and being rather constant as a function of the power of the pump beam.
\end{abstract}

\section{Introduction}

Research in the field of nanostructures has found a source of exciting new phenomena in the case of plasmonic nanofluids and colloids of noble metal nanoparticles, leading to an increasing number of applications, such as theranostics [1], biosensors [2], imaging and spectroscopy [3] and coolants [4], and phototherapy [5]. These applications take advantage of both the special optical and the thermal transport properties of the nanoparticles that arise from the nanoscale dimension of the medium and the high surface-to-volume ratio.

For applications based on the thermal transport of nanofluids, the knowledge of the thermal diffusivity $(D)$ is fundamental which is a material-specific property and measures the rate of heat transfer under unsteady conditions [6]. The thermal diffusivity is defined as $D=\kappa / \rho c_{p}$, where $\kappa$ is the thermal conductivity, $\rho$ is the mass density, and $c_{p}$ is the specific heat capacity. Besides the numerous studies employing techniques that allow direct access to the thermal conductivity and heat capacity of nanofluids, there are only a small number of studies that employ experimental techniques to directly obtain the thermal diffusivity of nanofluids. Some available methods and techniques are laser flash [7], hot-wire $[8,9]$, photoacoustic [10-12], photopyroelectric [13], thermalwave resonator cavity [14], and the thermal lens technique [15]. The last one is based on the optical properties of a medium with optical absorption and has been employed previously to obtain the thermal diffusivity of colloids of superparamagnetic nanoparticles [16]. Noble metal nanoparticles exhibit a strong optical absorption in the visible range of the electromagnetic spectrum due to the existence of a resonance of the localized surface plasmon (LSP), a nonpropagating collective oscillation mode of the conduction electrons of metallic nanostructures [17]. In the case of Ag nanoparticles, the maximum absorbance due to the LSP is at frequencies corresponding to the blue region of the visible spectrum. The details of the absorption band depend on the size and shape of the nanoparticle as well as the aggregation state: the bigger the nanoparticle or the aggregate, the bigger the redshift [18]. Previous works that have reported the thermal diffusivity of AgNF employing the thermal lens technique considered silver nanoparticles (AgNPs) produced by radiation $[19,20]$ and laser ablation [21]. Besides the thermal lens approach, others techniques such as microflash [22] and the photopyroelectric technique [23] have been employed to determine 


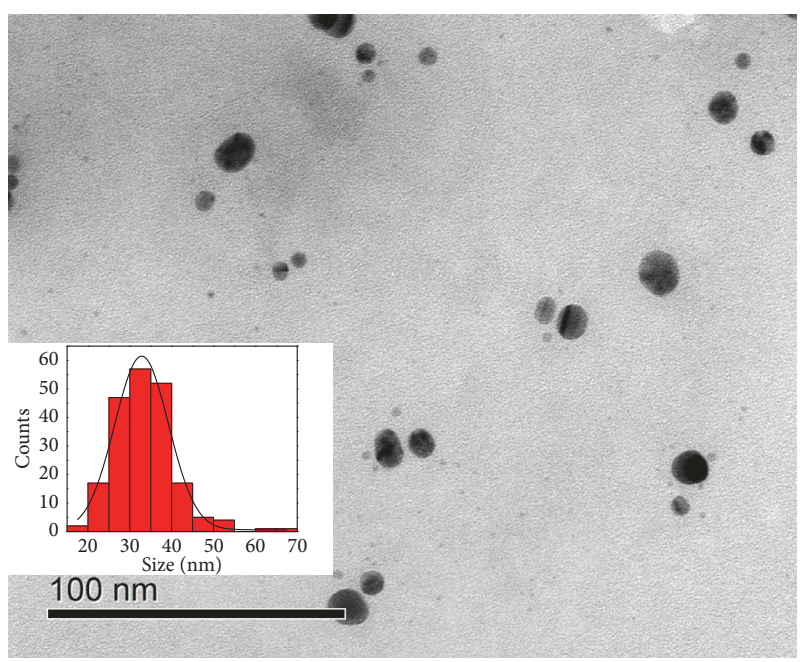

FIgURE 1: Image taken using TEM of the AgNPs and particle size histogram. Mean size: $32 \pm 10 \mathrm{~nm}$.

$D$ in AgNFs. Envisaging applications of the nanofluids as cooling fluids or in medicine, it is desirable to employ fluids with low viscosity. It is also know that the viscosity of nanofluids is an increasing function of the nanoparticle concentration [24] and becoming non-Newtonian at high concentrations in some cases [25]. Many of the previous mentioned techniques actually employ highly concentrated colloids due to limitations of the technique. Such limitations are not present in the thermal lens technique, allowing us to obtain the diffusivity of highly diluted colloids. In this paper, we report on research of the thermal diffusivity of highly diluted silver nanocolloids produced by a chemical reduction approach adapted from Turkevich method, by using the time-resolved mode-mismatched dual-beam thermal lens technique.

\section{Materials and Methods}

PVP-coated AgNPs (AgNP@PVP) were synthesized by adapting the chemical reduction method proposed by Turkevich [26] to produce Au nanoparticles [22]. In this bottom-up method, $75 \mathrm{~mL}$ of a $1 \mathrm{mM}$ solution of silver nitrate $\left(\mathrm{AgNO}_{3}\right.$, Synth PA) is heated to boiling point under magnetic stirring. Next, $7.5 \mathrm{~mL}$ of a $10 \mathrm{mM}$ solution of sodium citrate dihydrate $\left(\mathrm{HOC}(\mathrm{COONa})\left(\mathrm{CH}_{2} \mathrm{COONa}\right)_{2} \cdot 2 \mathrm{H}_{2} \mathrm{O}\right)$, Synth $\left.\left.\mathrm{PA}\right)\right)$ is added and after 4 minutes, $2 \%$ by mass of polyvinylpyrrolidone (PVP), a polar polymer that improves the stability of the colloid is also added. The concentration of AgNPs in the stock colloid was calculated to be $c_{s}=(1.54 \pm 0.05) \times 10^{12} \mathrm{~mL}^{-1}$ or approximately $10^{-7} \mathrm{vol} \%$. From the stock nanofluid, samples of concentration $f c_{s}$ were obtained, where $f=$ $1 / 2,1 / 3,1 / 4, \cdots$, by diluting aliquots of the stock colloid $(f=1)$ with double distilled water. Figure 1 shows an image, taken using transmission electron microscopy (TEM), of the synthesized AgNPs, which have a mean size of about $32 \pm$ $10 \mathrm{~nm}$. The UV-Vis spectrum for one of the diluted samples $(f=0.1)$ is shown in Figure 2. The spectrum displays the

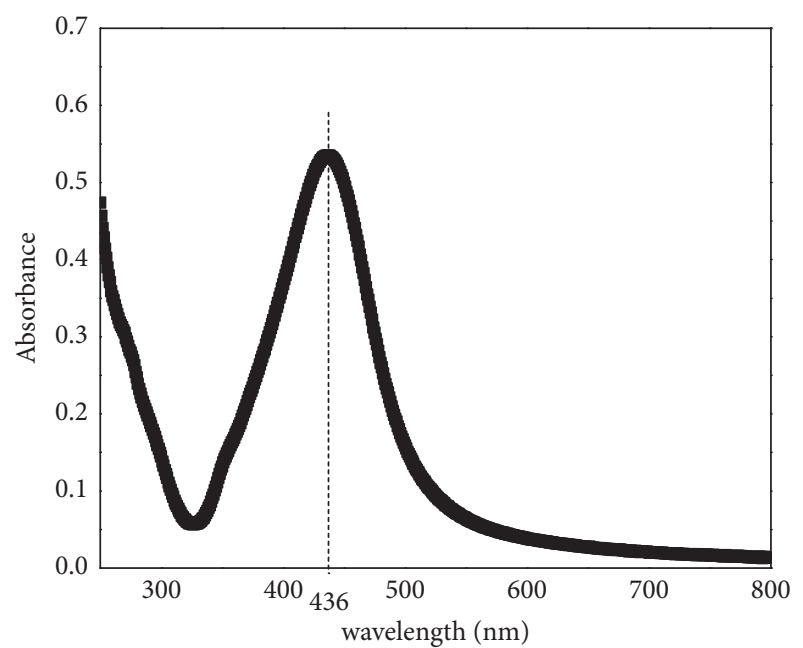

FIgURE 2: UV-Vis spectrum of the AgNPs, displaying the LSP resonance with a maximum at $436 \mathrm{~nm}$.

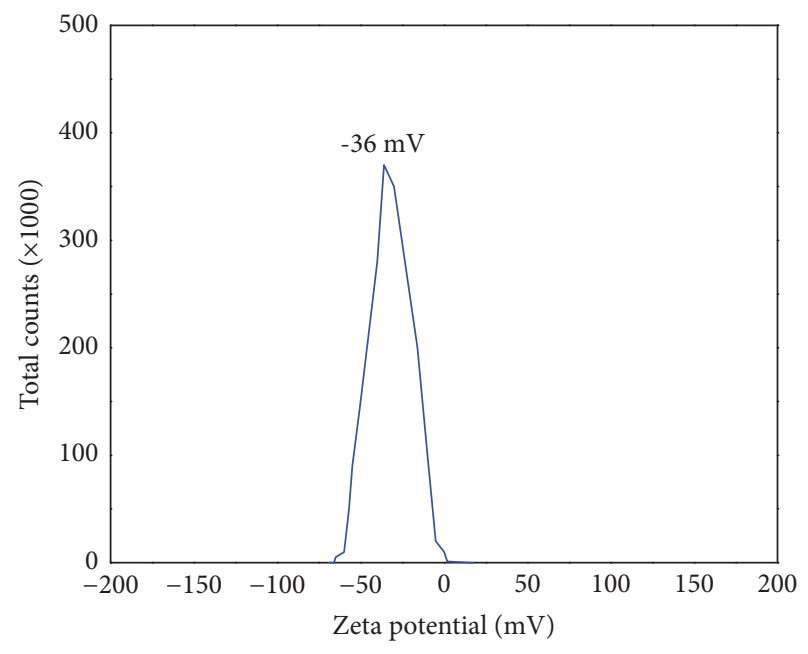

FIgURE 3: Zeta potential of the PVP@AgNPs.

typical peak of the LSP resonance of metallic nanoparticles, with a maximum at $436 \mathrm{~nm}$. The spectrum also indicates that the dilution does not lead to aggregation of the nanoparticles, which would be evident by a strong redshift and broadening of the peak. The stability of the nanoparticles was determined by the measurement of the zeta potential (Zetasizer Nano ZS90, Malvern) and this is shown in Figure 3. The value of $-36 \mathrm{mV}$ indicates that the nanoparticles have a good stability.

The photothermal technique employed in this work to measure the thermal diffusivity is the time-resolved modemismatched dual-beam thermal lens technique, which is based in the thermal lens effect. This effect consists of inducing a lens-like element in the sample following a nonradiative decay process due to an optical excitation of the absorbing medium. Pulses of second-long duration of a continuous wave $(\mathrm{CW})$ single-mode $\mathrm{TEM}_{00}$ laser beam (pump laser), obtained by a mechanical shutter, are used to excite the sample and to induce a thermal lens, which is then probed by a second CW single-mode $\mathrm{TEM}_{00}$ laser (probe 


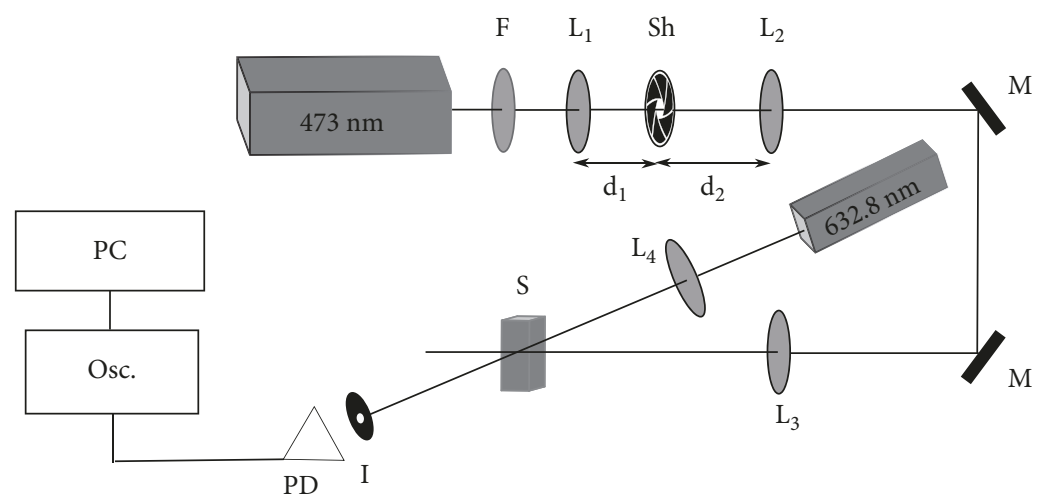

FIGURE 4: Sketch of the mode-mismatched dual-beam thermal lens technique: lens (L), mirror (M), sample (S), shutter (Sh), iris (I), IR filter $(\mathrm{F})$, and photodetector $(\mathrm{PD}) . d_{i}(i=1,2)$ represents the focal distance.

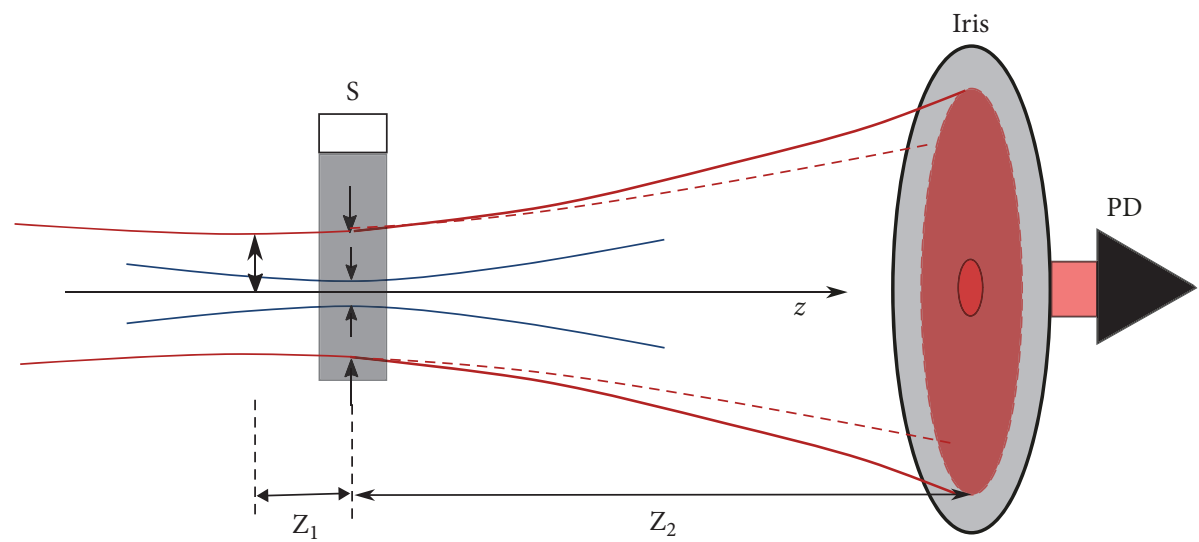

Figure 5: Representation of the positions of the laser beams in the sample for the two-beam thermal lens experimental setup. PD: photodetector. Dotted red line represents the trajectory of the probe beam without the thermal lens effect. Solid red line represents the actual trajectory of the probe beam.

laser). A sketch of the mode-mismatched dual-beam thermal lens technique apparatus is shown in Figure 4. The shutter is positioned at the common focal point of the set of lenses $\mathrm{L}_{1}$ and $\mathrm{L}_{2}$, as shown in Figure 4, to reduce the time of onset of the first half-cycle.

The probe beam propagates along the $z$-direction. A photodetector is used to monitor the temporal dependence of the transmitted probe-beam intensity $S(t)$, in the far field and at the center of the probe beam. The normalized signal $\Gamma(t)=S(t) / S(0)$, where $S(0)$ is the signal of the photodetector at $t=0$, when the thermal lens has not been formed yet, can be written as $[27,28]$

$$
\begin{aligned}
& \Gamma(t)=\left\{1-\frac{\theta}{2}\right. \\
&\left.\cdot \tan ^{-1}\left(\frac{2 M V}{\left[(1+2 M)^{2}+V^{2}\right]\left(t_{c} / 2 t\right)+1+2 M+V^{2}}\right)\right\}^{2},
\end{aligned}
$$

where

$$
V=\frac{Z_{1}}{Z_{c}}+\frac{Z_{c}}{Z_{2}}\left[\left(1+\frac{Z_{1}}{Z_{c}}\right)^{2}\right]
$$

$$
\begin{aligned}
\theta & =-\frac{P_{e} \alpha_{o} L_{e f f}}{\kappa \lambda_{p}}\left(\frac{d n}{d T}\right) ; \\
M & =\left(\frac{w_{1 p}}{w_{o e}}\right)^{2} ;
\end{aligned}
$$

Here, $\alpha_{o}$ is the linear optical absorption coefficient, $P_{e}$ is the excitation beam power, $\lambda_{p}$ is the probe laser wavelength, $L_{e f f}=1-e^{-\alpha_{0} L} / \alpha_{0}$ is the effective thickness of a sample of thickness $L, Z_{c}=\pi w^{2} / \lambda$ is the confocal distance, $d n / d T$ is the thermo-optic coefficient or variation of the refractive index of sample with temperature, $w_{o i}(i=p, e)$ is the minimum radius of the probe and the excitation beam, respectively, $w_{1 p}$ is the beam radius of the probe beam inside the sample, and $Z_{i}(i=1,2)$ are the distances of the sample to the position of the minimum radius of the probe beam and the iris, respectively, and $t_{c}$ is the characteristic thermal time constant. Figure 5 shows a schematic diagram of the laser beams inside the sample contained in the quartz cell.

The effective thermal diffusivity of the medium is given by

$$
D=\frac{w_{o e}^{2}}{4 t_{c}} .
$$




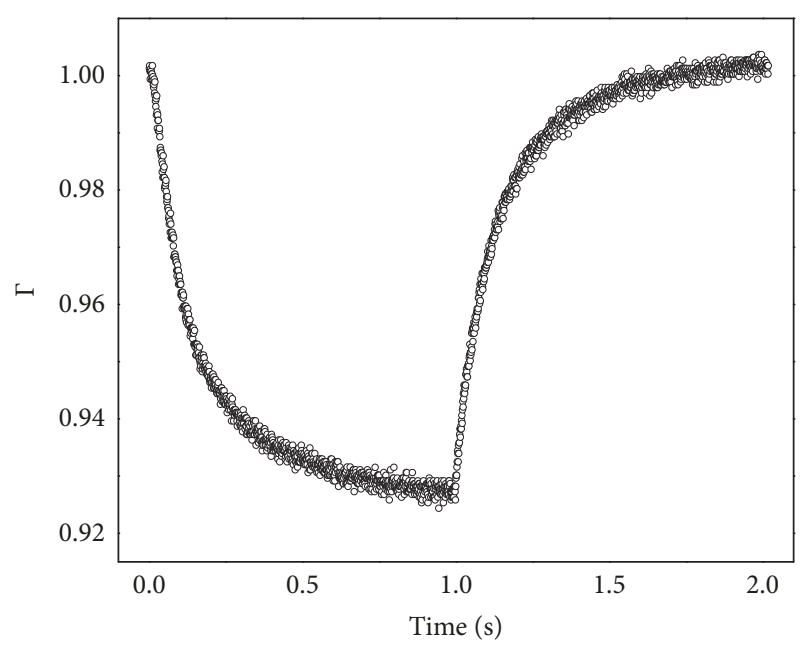

FIgURE 6: Typical normalized transmittance in a cycle ( $\mathrm{T}=2 \mathrm{~s})$ of the shutter for a sample of the stock colloid and a pump beam of $1 \mathrm{~mW}$.

Parameters $\theta$ and $t_{c}$ were obtained by fitting the normalized thermal lens signal as a function of time ( $\Gamma$ ) to (1). Finally, the thermal diffusivity can be calculated from (5).

In our experimental setup, the excitation and probe beams have wavelengths of $473 \mathrm{~nm}$ (Ciel, Laser Quantum) and $632.8 \mathrm{~nm}$ (HeNe laser, Melles Griot), whose beam waists are $w_{o e}=20.4 \mu \mathrm{m}$ and $w_{o p}=37.5 \mu \mathrm{m}$, respectively. The geometrical parameters of the setup are $M=47.929, V=$ $6.01, Z_{1}=5.1 \mathrm{~cm}, Z_{2}=382 \mathrm{~cm}$, and $w_{1 p}=338.3 \mu \mathrm{m}$, and the angle between both beams is about $1^{\circ}$. Our experimental setup also includes an IR filter to remove any IR radiation from the pump laser. The sample was placed in a quartz cell of $0.5 \mathrm{~cm}$ optical path length and all measurements were made at room temperature $\left(25^{\circ} \mathrm{C}\right)$.

Before each measurement, the sample was placed in an ultrasonic bath for 30 minutes for homogenization.

\section{Experimental Results}

Figure 6 shows a typical normalized transmittance over 1 cycle (opened-closed) of the shutter, whose period is $\mathrm{T}=2$ s, for a sample of the stock colloid and Figure 7 shows the normalized transmittance just after the opening of the shutter for a sample of the stock colloid for different powers of the pump beam. The higher the power of the pump laser, the bigger the value of $\theta$ (3) and the bigger the variation of the amplitude of the normalized transmittance, as predicted by (1).

The decreasing signal in the first half of the cycle just after the opening of the shutter and for the geometrical configuration of the experimental setup (sample after the focus of the probe laser), is the finger-print of an induced negative or divergent lens. On the other hand, the characteristic time constant for the emergence of the induced lens in the samples is on a millisecond (ms) time scale, which is expected from the photothermal effect. In the second half of the cycle, the sample relaxes via a nonradiative process

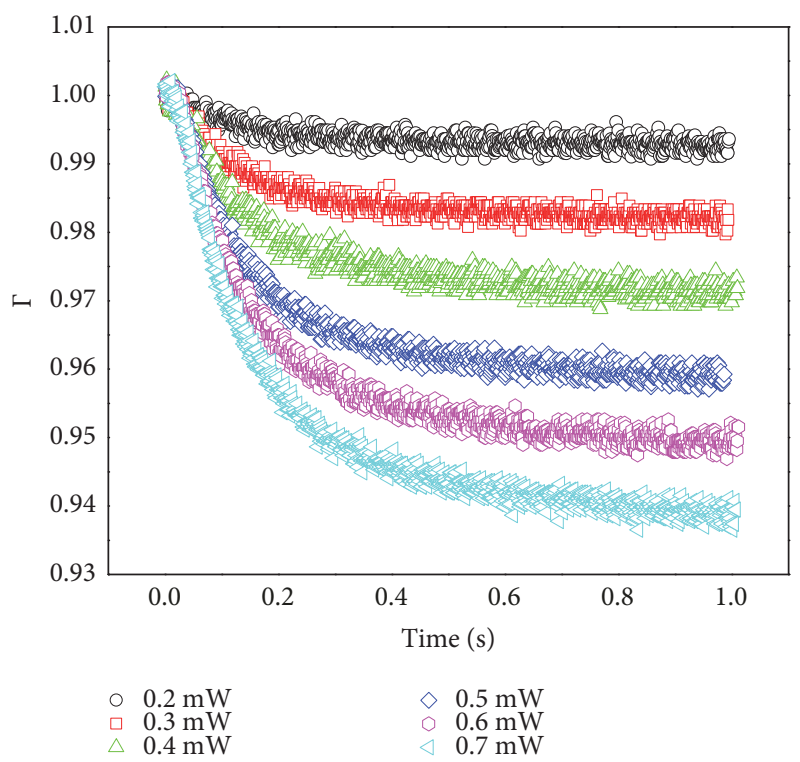

FIGURE 7: Normalized transmittance for a sample of stock colloid as a function of the power of the pump beam.

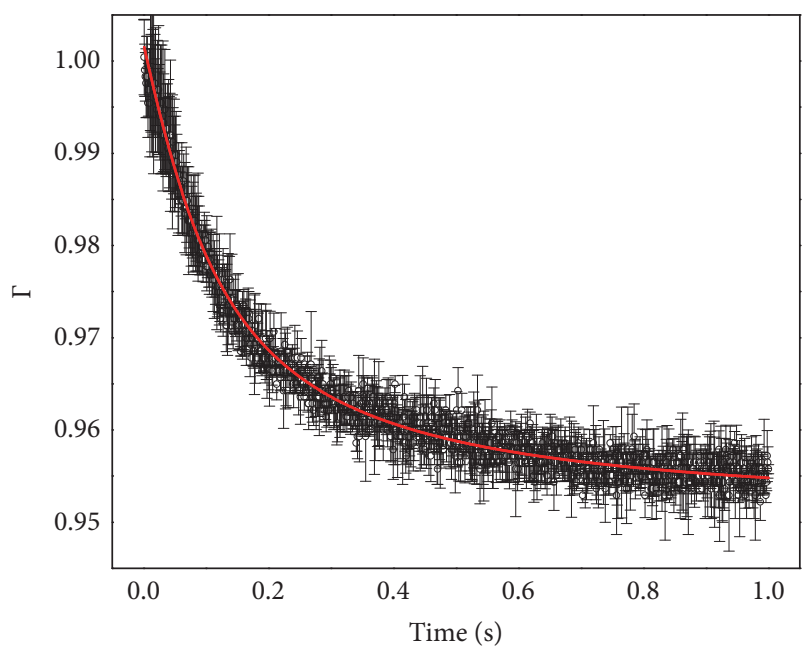

FIgURE 8: Red line shows typical fit to (1) of the normalized transmittance in the first half of the cycle.

to room temperature. Similar curves were obtained for all diluted colloids and pure water.

Figure 8 shows a typical fit of the normalized transmittance in the first half of the cycle to (1). The error bars represent the standard deviation of 45 measurements.

To test the precision of our setup, measurements of the thermal diffusivity of the pure double distilled water used to prepare and dilute the stock colloid, $D_{m}$ were taken. The value obtained was $D_{m}=(1.42 \pm 0.15) \times 10^{-7} \mathrm{~m}^{2} / \mathrm{s}$, which is close to the values previously reported for water $[6,29$, 30]. Figure 9 shows the value of the thermal diffusivity for increasing values of the power of the pump beam for a sample corresponding to $f=1 / 3$. The thermal diffusivity is almost constant, with a mean value of $D=1.74 \pm 0.03 \mathrm{~m}^{2} / \mathrm{s}$, at 


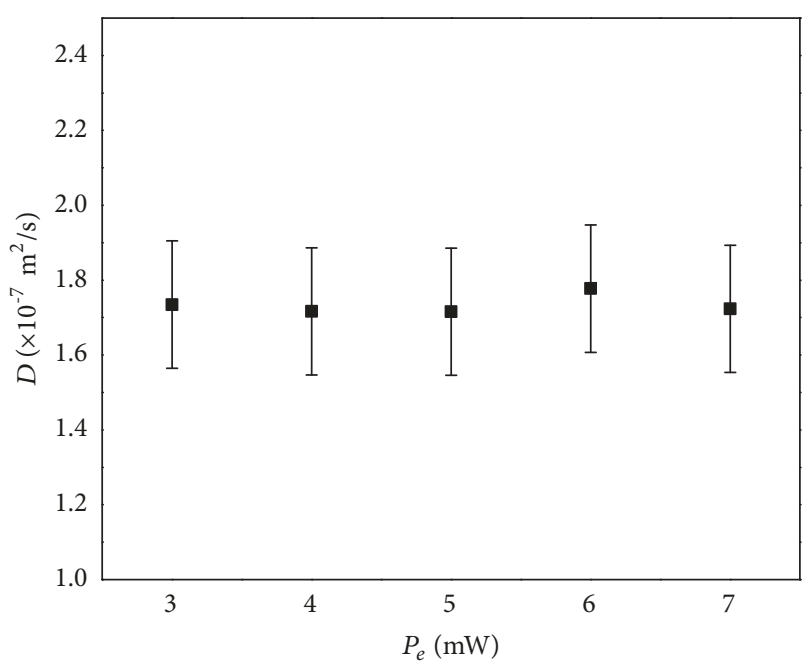

FIgURE 9: Thermal diffusivity as a function of the power of the pump beam for sample corresponding to $f=1 / 3$.

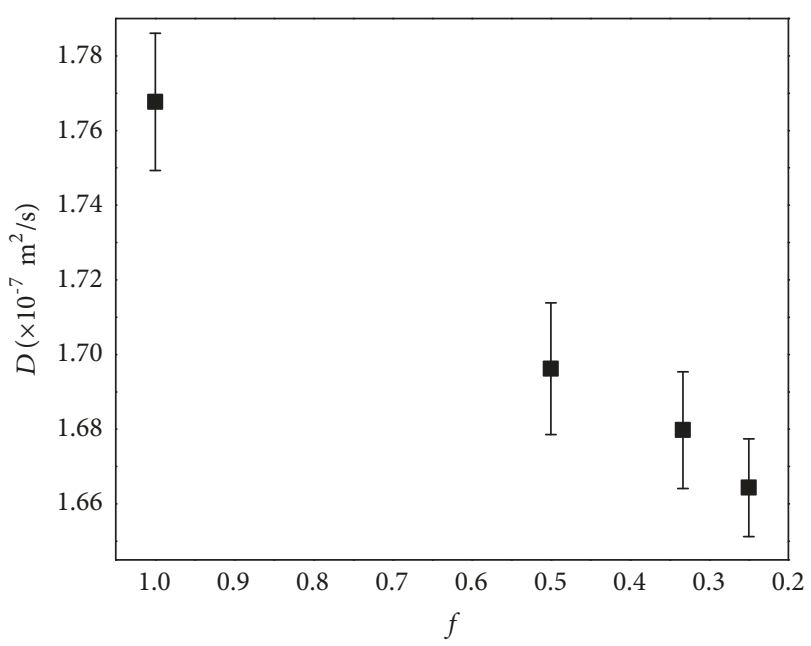

Figure 10: Normalized diffusivity of the diluted $(f<1)$ and stock $(f=1)$ colloids.

least for this power range, showing that no other diffusive process is concomitant with the thermal diffusion process. Our results also show that the thermal lens technique is more suitable for determining the thermal diffusivity of a colloid at extremely low concentrations than the laser flash method [22]. Figure 10 shows the values of the thermal diffusion coefficients for the samples as a function of the concentration of AgNPs. As can be seen, the diffusivity of the colloid decreases on increasing the dilution, that is, diminishing the value of $f$. It is worth noting that even at such a low concentration as that corresponding to $f=0.25$, the diffusivity is about $17 \%$ higher than that of pure water. The same trend was observed in highly concentrated dilutions of AgNPs in organic fluids [8]. The decrease of the thermal diffusivity with the dilution of the nanofluid can be understood taking into account the dependence of the thermal conductivity on the filling factor. For low concentrations the thermal conductivity of a nanofluid is directly proportional to the fill factor [31].

\section{Conclusions}

In summary, we have measured the thermal diffusivity of diluted colloids of PVP-coated AgNPs, produced by chemical reduction, by employing the time resolved modemismatched dual beam thermal lens technique, with an excitation beam whose frequency is close to the surface plasmon resonance of the AgNPs. The thermal diffusivity of the samples shows a strong enhancement when compared to the value for double distilled water, being relatively constant at low powers of the pump beam. Also, the technique shows a superior sensitivity in determining the thermal diffusivity when compared to alternative techniques.

\section{Data Availability}

The data used to support the findings of this study are available from the corresponding author upon request.

\section{Conflicts of Interest}

No potential conflicts of interest were reported by the authors.

\section{Acknowledgments}

The authors acknowledge the National Institute of Science and Technology Complex Fluids (INCT-FCx) and the Brazilian agencies CNPq, CAPES, Fundação Araucária, and Secretaria de Ciência, Tecnologia e Ensino Superior do Paraná, for financial support.

\section{References}

[1] P. di Pietro, G. Strano, L. Zuccarello, and C. Satriano, "Gold and silver nanoparticles for applications in theranostics," Current Topics in Medicinal Chemistry, vol. 16, no. 27, pp. 3069-3102, 2016.

[2] H. Malekzad, P. Sahandi Zangabad, H. Mirshekari, M. Karimi, and M. R. Hamblin, "Noble metal nanoparticles in biosensors: Recent studies and applications," Nanotechnology Reviews, vol. 6, no. 3, pp. 301-329, 2017.

[3] A. Biswas, T. Wang, and A. S. Biris, "Single metal nanoparticle spectroscopy: optical characterization of individual nanosystems for biomedical applications," Nanoscale, vol. 2, no. 9, pp. 1560-1572, 2010.

[4] H. B. Kulkarni, M. M. Nadakatti, M. S. Patil, and R. M. Kulkarni, "A review on nanofluids for machining," Current Molecular Pharmacology, vol. 13, no. 6, pp. 634-653, 2017.

[5] C. Yao, L. Zhang, J. Wang et al., "Gold Nanoparticle Mediated Phototherapy for Cancer," Journal of Nanomaterials, vol. 2016, Article ID 5497136, 29 pages, 2016.

[6] A. Salazar, "On thermal diffusivity," European Journal of Physics, vol. 24, no. 4, pp. 351-358, 2003.

[7] Y. Yang, A. Oztekin, S. Neti, and S. Mohapatra, "Particle agglomeration and properties of nanofluids," Journal of Nanoparticle Research, vol. 14, no. 5, 2012. 
[8] S. M. S. Murshed, K. C. Leong, and C. Yang, "Determination of the effective thermal diffusivity of nanofluids by the double hot-wire technique," Journal of Physics D: Applied Physics, vol. 39, no. 24, pp. 5316-5322, 2006.

[9] X. Zhang, H. Gu, and M. Fujii, "Experimental study on the effective thermal conductivity and thermal diffusivity of nanofluids," International Journal of Thermophysics, vol. 27, no. 2, pp. 569-580, 2006.

[10] T. A. El-Brolossy and O. Saber, "Non-intrusive method for thermal properties measurement of nanofluids," Experimental Thermal and Fluid Science, vol. 44, pp. 498-503, 2013.

[11] F. Agresti, A. Ferrario, S. Boldrini et al., "Temperature controlled photoacoustic device for thermal diffusivity measurements of liquids and nanofluids," Thermochimica Acta, vol. 619, pp. 48-52, 2015.

[12] G. A. López-Muñoz, J. A. Pescador-Rojas, J. Ortega-Lopez, J. S. Salazar, and J. Abraham Balderas-López, "Thermal diffusivity measurement of spherical gold nanofluids of different sizes/concentrations," Nanoscale Research Letters, vol. 7, article 423, 2012.

[13] M. Noroozi and A. Zakaria, "Measuring Nanofluid Thermal Diffusivity and Thermal Effusivity: The Reliability of the Photopyroelectric Technique," in Nanofluid Heat and Mass Transfer in Engineering Problems, Chapt. 4, M. S. Kandelousi, Ed., IntechOpen, 2017.

[14] M. Noroozi, S. Radiman, A. Zakaria et al., "Optical-fiber thermal-wave-cavity technique to study thermal properties of silver/clay nanofluids," Journal of the European Optical Society: Rapid Publications, vol. 9, Article ID 14046, 2014.

[15] E. Shahriari, W. M. Yunus, and R. Zamiri, "The effect of nanoparticle size on thermal diffusivity of gold nano-fluid measured using thermal lens technique," Journal of the European Optical Society: Rapid Publications, vol. 8, Article ID 13026, 2013.

[16] V. M. Lenart, N. G. Astrath, R. F. Turchiello, G. F. Goya, and S. L. Gómez, "Thermal diffusivity of ferrofluids as a function of particle size determined using the mode-mismatched dualbeam thermal lens technique," Journal of Applied Physics, vol. 123, no. 8, Article ID 085107, 2018.

[17] S. A. Maier, Plasmonics: Fundamentals and Applications, Springer, New York, NY, USA, 2007.

[18] O. Kvítek, J. Siegel, V. Hnatowicz, and V. Švorčík, "Noble Metal Nanostructures Influence of Structure and Environment on Their Optical Properties," Journal of Nanomaterials, vol. 2013, Article ID 743684, 15 pages, 2013.

[19] R. Zamiri, B. Z. Azmi, E. Shahriari et al., "Thermal diffusivity measurement of silver nanofluid by thermal lens technique," Journal of Laser Applications, vol. 23, no. 4, 2011.

[20] E. Shahriari, M. G. Varnamkhasti, and R. Zamiri, "Characterization of thermal diffusivity and optical properties of Ag nanoparticles," Optik - International Journal for Light and Electron Optics, vol. 126, no. 19, pp. 2104-2107, 2015.

[21] L. Rodriguez, J. F. Cárdenas-García, and C. C. Vera, "Measurement of thermal diffusivities of silver nanoparticle colloidal suspensions by means of a frequency-resolved thermal lensing approach," Optics Expresss, vol. 39, no. 12, pp. 3406-3409, 2014.

[22] F. Agresti, S. Barison, S. Battiston et al., "Tuning the thermal diffusivity of silver based nanofluids by controlling nanoparticle aggregation," Nanotechnology, vol. 24, no. 36, Article ID 365601, 2013.

[23] M. Noroozi, S. Radiman, A. Zakaria, and S. Soltaninejad, "Fabrication, characterization, and thermal property evaluation of silver nanofluids," Nanoscale Research Letters, vol. 9, pp. 1-10, 2014.

[24] L. Godson, B. Raja, D. M. Lal, and S. Wongwises, "Experimental investigation on the thermal conductivity and viscosity of silverdeionized water nanofluid," Experimental Heat Transfer, vol. 23, no. 4, pp. 317-332, 2010.

[25] A. Minakov, V. Rudyak, and M. Pryazhnikov, "Rheological behavior of water and ethylene glycol based nanofluids containing oxide nanoparticles," Colloids and Surfaces A: Physicochemical and Engineering Aspects, vol. 554, pp. 279-285, 2018.

[26] J. Turkevich, P. C. Stevenson, and J. Hillier, "A study of the nucleation and growth processes in the synthesis of colloidal gold," Discussions of the Faraday Society, vol. 11, pp. 55-75, 1951.

[27] M. L. Baesso, J. R. D. Pereira, A. C. Bento, A. J. Palangana, A. M. Mansanares, and L. R. Evangelista, "Thermal lens spectrometry to study complex fluids," Brazilian Journal of Physics, vol. 28, no. 4, pp. 359-368, 1998.

[28] J. Shen, R. D. Lowe, and R. D. Snook, "A model for cw laser induced mode-mismatched dual-beam thermal lens spectrometry," Chemical Physics, vol. 165, no. 2-3, pp. 385-396, 1992.

[29] D. W. James, "The thermal diffusivity of ice and water between -40 and $+60 \circ$ C," Journal of Materials Science, vol. 3, no. 5, pp. 540-543, 1968.

[30] P. R. B. Pedreira, L. Hirsch, J. R. D. Pereira, A. N. Medina, A. C. Bento, and M. L. Baesso, "Temperature dependence of the thermo-optical properties of water determined by thermal lens spectrometry," Review of Scientific Instruments, vol. 74, no. 1, pp. 808-810, 2003.

[31] D. H. Kumar, H. E. Patel, V. R. R. Kumar, T. Sundararajan, T. Pradeep, and S. K. Das, "Model for heat conduction in nanofluids," Physical Review Letters, vol. 93, no. 14, Article ID 144301, 2004. 

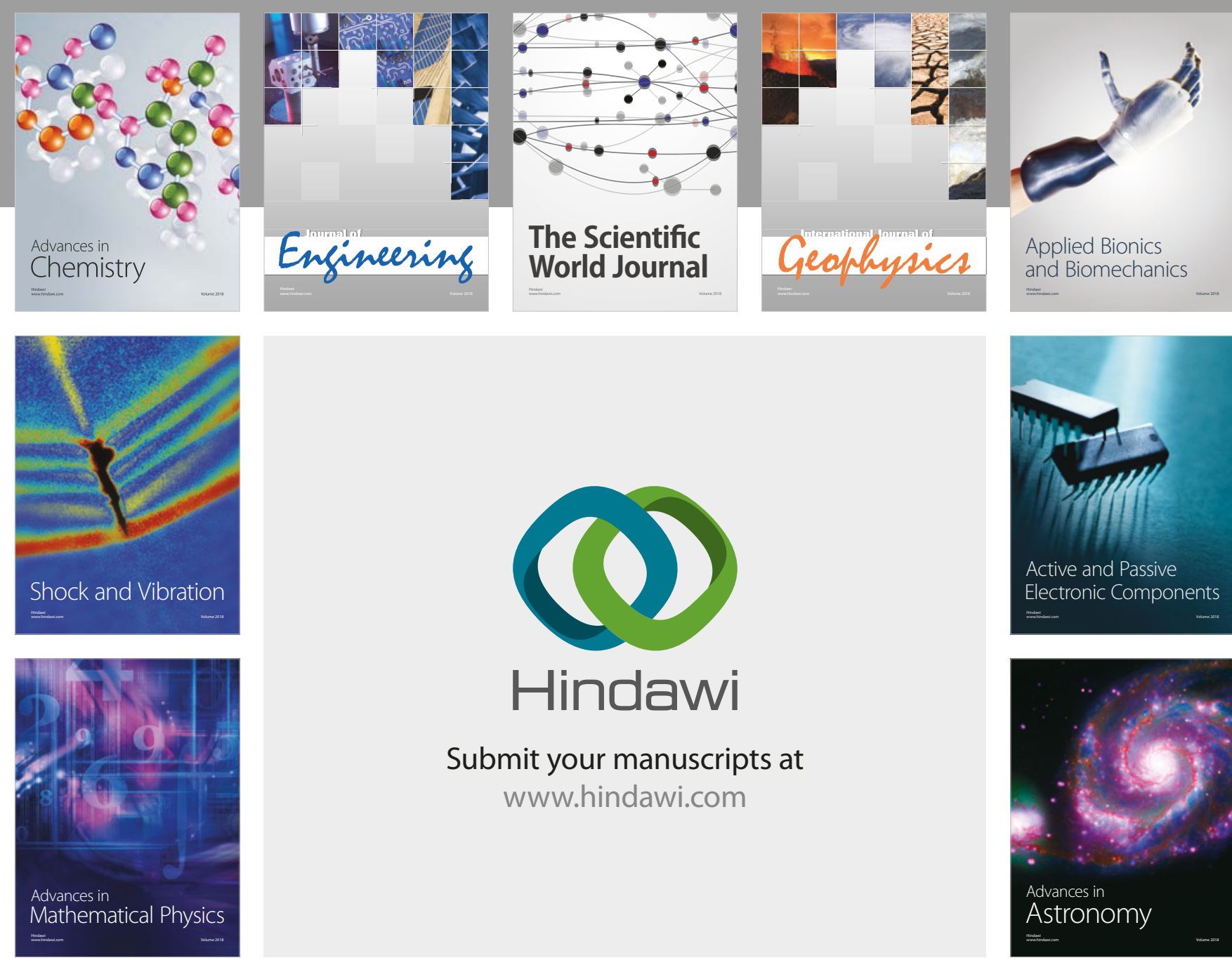

Submit your manuscripts at

www.hindawi.com

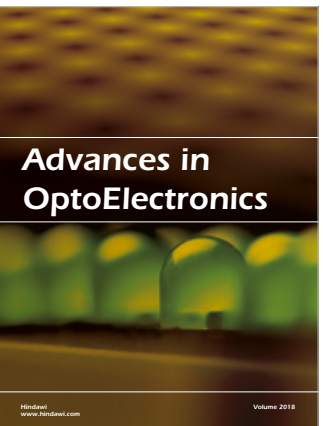

\section{Rotcting Machinery}
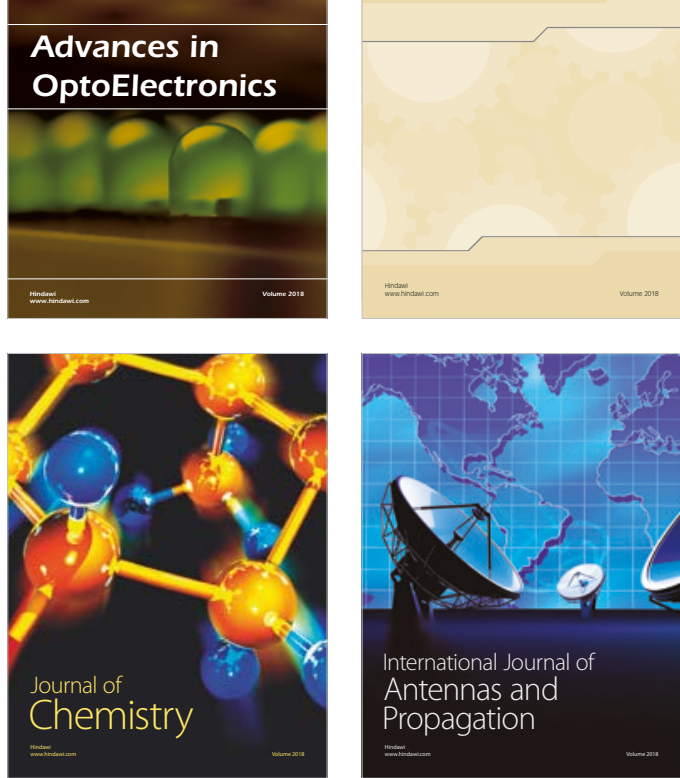

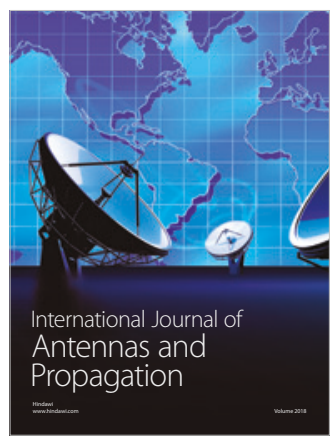

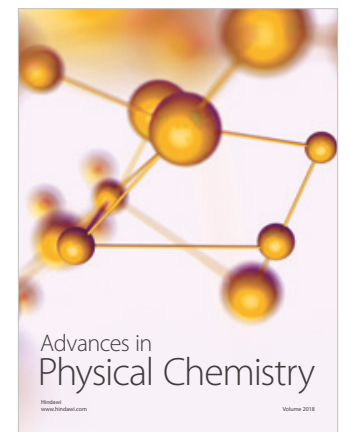

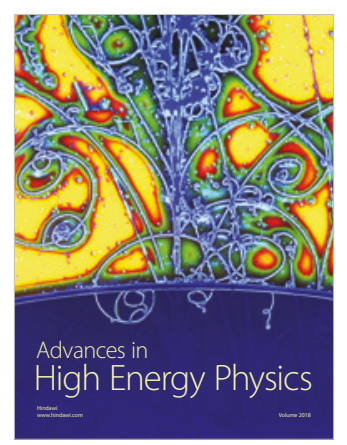

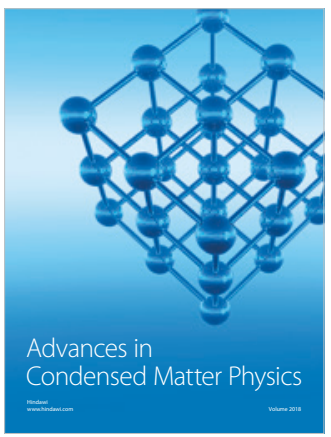

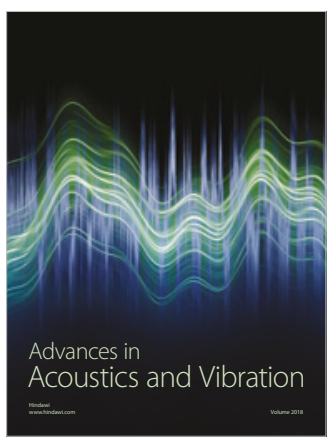

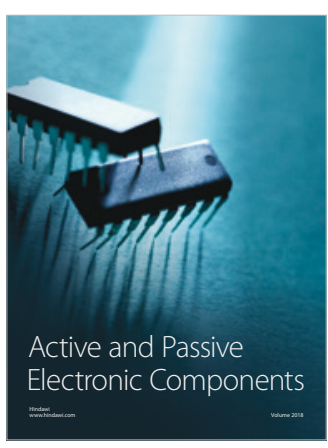
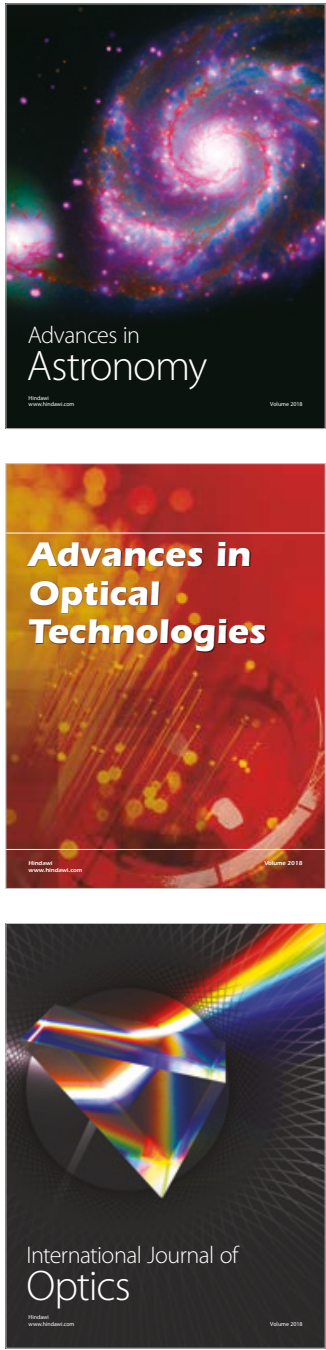